\title{
A COMPREHENSIVE STUDYON APPLICATION OF DEEP LEARNING IN BRAIN TUMOR DETECTION
}

\author{
Josmy Mathew, Research Scholar, Sathyabama University, Chennai \\ Dr. N. Srinivasan, Adjunct Professor, BITS Pilani, Chennai Campus
}

\begin{abstract}
Deep Learning is an area of machine learning which, because of its capability to handle a large quantity of data, has demonstrated amazing achievements in each field, notably in biomedicine. Its potential and abilities were evaluated and utilised with effective prognosis in the identification of brain tumours with MRI pictures. The diagnosis of MRI images by computer-aided brain tumours includes tumour identification, segmentation and classification. Many researches have concentrated in recent years on conventional or basic machine learning approaches in the detection of brain tumours. Throughout this overview, we offer a comprehensive assessment of the surveys that have been reported so far and the current approaches for detecting tumours. Our review examines the major processes in deep learning approaches for detecting brain tumours including preprocessing, extraction of features and classification and their performance and limitations. We also explore state-ofthe-art neural network models to identify brain tumours through extensive trials with and without data augmentation. This review also discusses existing data sets for brain tumour detection assessments.
\end{abstract}

Keywords-Deep learning, brain tumor, classification, feature extraction.

\section{Introduction}

A complicated human body organ is the brain. Inappropriate mitotic process influences the activity of morphological cells in the human brain. Tumor cells with varied morphological features such as aggressiveness and size are developed throughout this phase. In terms of grades, the brain tumour comprises two wide groups. These are slow-growing and aggressive tumours of inferior quality. The high-level tumour produces blood-brain blockage and the ejection of the antigen. Most malignant tumours in the brain are therefore nominated for neuroepithelial malignancies. Glioblastoma is the most frequent kind of tumour. Only 5\% of tumour patients are able to survive for a maximum period of 05 years or less.

In comparison with nearby cells, tumour cells typically exhibit a low contrast. Accurate brain tumour identification is therefore a crucial issue. Mostly, MRI method is a painless test to identify brain tumour and helps to examine the location of the tumour from different viewpoints. It is therefore regarded the finest way of diagnosing and analysing all forms of brain tumours. The last several decades of the processing of images and computer vision assisted humanity with automatic diagnostic methods to identify various illnesses. In many diagnostic procedures these processes in the medical sector have previously been supported by medical professionals and specialists.

Cancer is seen as a threat to civilization because of its deadly character amongst all dangerous diseases. An expert traditionally evaluates medical pictures and determines the likelihood of growing a tumour manually. Manually identification of a tumour symptom and this decision is the method by which most doctors can avoid prescribing future medical 
therapies because of the deadly nature of the brain tumour. Computer vision techniques are the most advanced and safe means of evaluating medical pictures. It contains brief pictures using different applications with built-in tumour identification and classification techniques. These algorithms may also be modified to perform tumour segmentation that indicates how the contaminated parts are separated from healthy portions of the under observation medical picture.

In order to be successful and timely for a patient, early diagnosis and categorization of a brain tumour are essential. The visual processing cortex is believed to be restricted in its capacity to decide in magnetic resonance imaging between different degrees of grey (MRI). This produces computer-aided diagnosis or categorization of brain tumours, which is appropriate for helping radiologists to see and define tumour kinds. These automated techniques for identification, segmentation and categorization of brain tumours play a crucial role in supporting mankind by lowering the likelihood of operation. If radiologists get puzzled well about characteristics of a tumour or wish to examine it thoroughly, they will always be helped by such approaches.

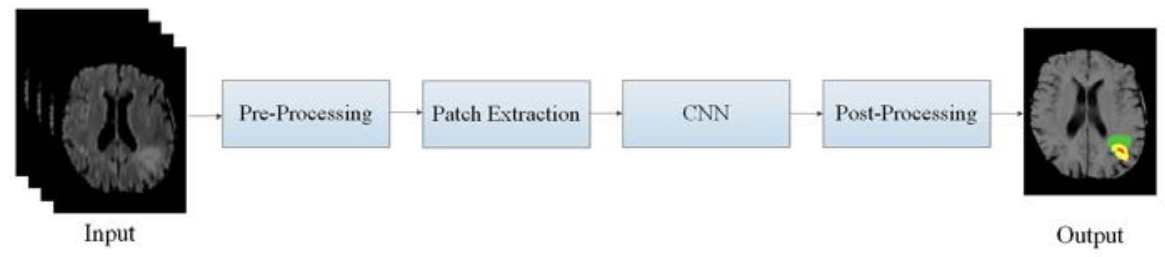

Figure 1. General Structure of Deep learning model in tumor detection

Image processors and computer vision experts are keen to provide accurate and effective techniques to identify, classify, and segregate malignancy automatically. The brain tumours consist of tissue that is improperly produced due to unchecked cell proliferation, and there is no physiologic function of this tissue inside the brain. Tumors not just to increase in brain size and pressure, but induce edoema, causing aberrant neurological signs. The International Brain Tumor Organization reports that the number of individuals who die as a result of brain tumours in affluent countries has grown $300 \%$. Tumors of the brain are categorised as metastatic tumours or primary brain tumours. Primary tumours originally include brain cells; however, cancer cells have migrated from another diseased part of the body into the brain in metastatic tumours.

Gliomas are presently the principal kind of tumour garnering the attention of tumour scientists. The concept of glioma describes many forms of glioma, from tumours known as glioblastoma multiforme to low-grade glioma such oligodendrogliomas or astrocytomas. Gliomas may be treated by chemotherapy, radiation, and surgery. The primary objective of the computerised diagnosis of brain tumours is to collect essential clinical information on the existence, location and type of tumour. The information gained by clinical imagery can guide, regulate and correctly diagnose and treat the tumour in future. These automated diagnostic approaches for brain tumours contain several methods that may be arranged in a pyramid.

Different strategies for preparing, selecting, marking and describing data are required for each phase of the pyramid. The early identification of tumours plays an important role in the improvement of treatment options. Techniques also provide coordinates, size, form and neurological condition for diagnosis include brain imaging techniques such as positron- 
emission tomography, a solitary energy density calculated radiography, ct scans, permanent magnet imagery, and magnetic-resonance spectroscopy. MRI offers extensive information on the architecture of human tissue and is regarded as a standard technology because of its broad availability and soft tissue contrast.

In order to create pictures of human tissues, the MRI utilises radio frequency waves with a strong magnetic field. The diagnosis of brain tumour comprises of the procedures of tumour identification, segmentation and classification. The MRI scans of tumours in a database, which is regarded as a fundamental and evident procedure, are mostly identified using brain tumour detection algorithms. However, approaches of brain tumour segmentation are utilised to locate and isolate distinct tumour tissues within MRI pictures. In addition, methods to identify aberrant pictures as malignant or benign tumour are utilised for the classification of brain tumours. These three hybrid approaches and methodologies provide radiologists with important information and help to interpret the MRI data needed for diagnosis. In the last several decades, numerous researchers have carried out important work on the diagnosis of brain tumours.
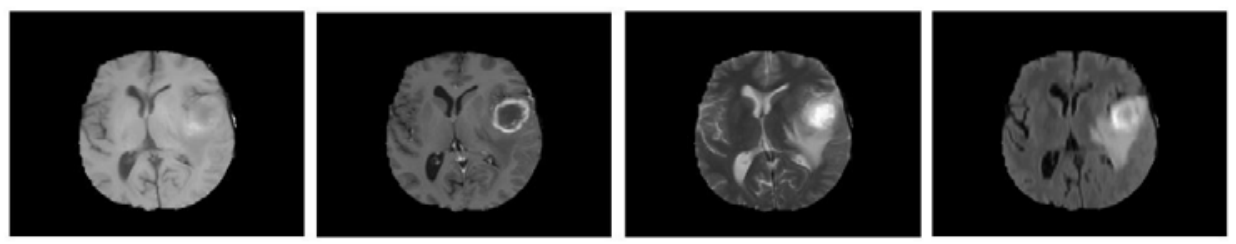

Figure 2. Sample brain MRI images

Segmentation of tumours and techniques of categorization were also suggested. The degree of monitoring and the ease of calculation depends on the clinical acceptability of diagnostic procedures. However, there are currently limited clinical applications; although considerable work is done, doctors still rely on the manual tumour projection, perhaps due to a lack of link between clinical scientists. Profound learning is an automated, semiautomatic and/or hybrid model that has been utilised frequently over the past few years to efficiently categorise the tumour and divide it in less time and with the greatest degree of exactness. This study evaluated recent research articles on the detection and classification of deep learning brain tumour.
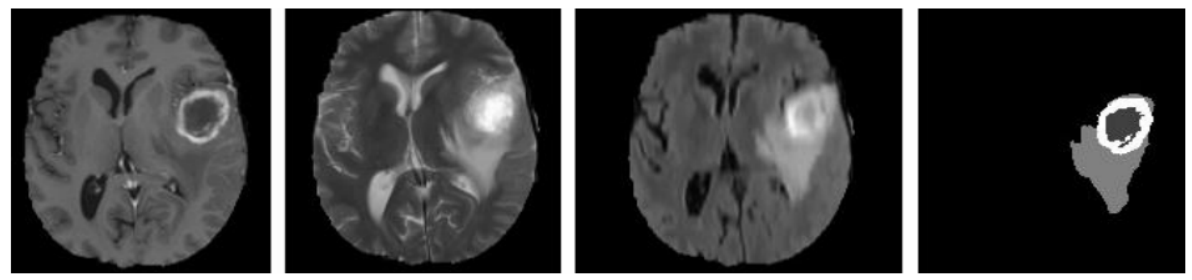

Figure 3. Segmented MRI image set

The latest research, both on detection and on categorization of brain tumours, was to be explored. The main reason why this type of review is carried out is that we plan on working on a particular multi-task deeper study model and want to explore the existing deep learning models to gain insight into the shortcomings and shortcomings that can be addressed through our proposed deep learning scheme. Multi-task learning architectures integrate many models to form a single model with a single learning process. Usually, these models have one source and several outputs. In the past five years, we have used both the journal and 
conference papers to write this review since, unexpectedly, many recently discovered profound learning models have been presented in conference presentations. We also found that in journals we published extremely comprehensive and intuitive evaluations which truly helped us to create our papers. Our survey can summarise the initial contributions as below:

Throughout this survey, we highlight their accomplishments and limits in all available CNN techniques for tumour identification. We study the whole tumour detection literature and identify its primary areas, research trends and niches as well as accessible benchmark data for testing.

- Inspired by the current successes of image classification deep learning models, we study and perfect multiple cognitive fatigue CNN models for tumour identification utilising two inputs and outputs with and without increase of data. We give extensive information on these models with several parameters for future study and diagnostic aid from different academics and radiologists.

- With the current growth in the utilisation of intelligent gadgets and cloud/negative computing in intelligent cities, these technologies are being used for health care systems. In this survey, we thus emphasise the individualised use by patients, distant experts, and medical centres of intelligent health services of tumour detection. This will make it easier to integrate into the present smart city ecosystem and more sustainable for its widespread use and acceptance.

- This review outlines the present problems in the field of tumour detection and provides a single summary of the entire profound literature based on learning. In addition, for further study in this field, we provide recommendations and future directions.

\section{Classification Models}

In this part, we review briefly the current literature's architectures and global methods. Tumor segmentation is the initial stage in the overall tumour identification pipeline, the main objective being current segmentation techniques. Tumor segmentation but at the other hand, other approaches have employed combined classification end-to-end models. Because the study focuses on tumour identification, we are only covering tumour detection procedures or those employing classification segmentation.

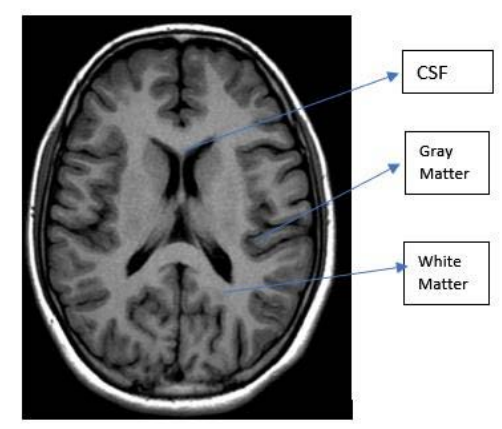

Figure 4. Normal brain MRI image

In the research examined, the main step before classification is different approaches for segmentation of tumours. For example, Akkus et al. categorised the brain tumour by 
means of three phases, for example, the registration of images with such a cubic b-spline smoothing approach, semi-automatic LGG software segmentation, and 1p/192 classification by CNN. The MRI data of 159 individuals are used using this technique, with 1p/19q confirmed status. To balance the distribution of data, several data augmentation approaches are utilised. Paul et al. have introduced two applications of machine learning for the diagnosis of brain cancers with increased data to improve their approach performance.

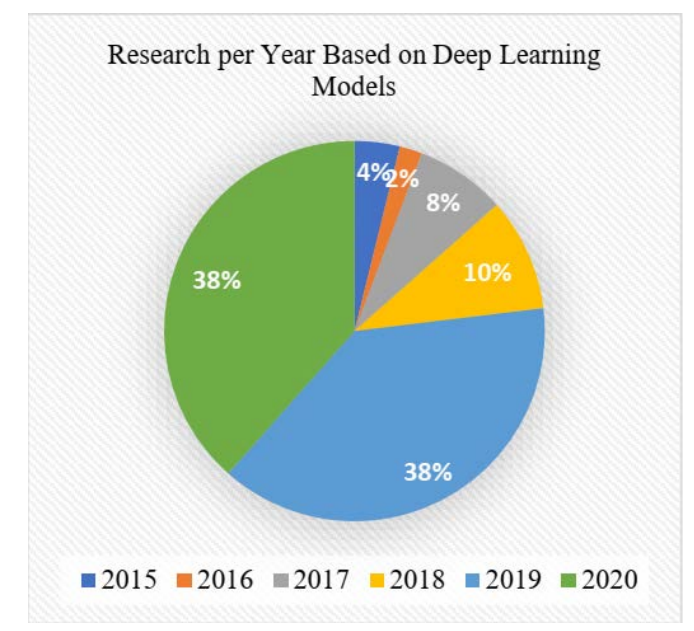

Figure 5. Deep learning model research distribution

The pretrained Multiforme AlexNet model for the identification and estimate of the life expectancy of patients with Multiforme Glioblastoma was also used by the company Ahmed et al. Balasooriya and Nawarathna have created their CNN for the identification of tumours. Their approach was evaluated by utilising a TCIA-data set split into five classes: astrocytoma, multi-form gliobastoma, oligodendroglioma, epithelial cells, and undetermined tumour. For 3-D brain pictures, Wong et al. presented a medical image grader. They removed functions from the M-Net segmentation network and transformed them into a pre-trained VGG-16 model for the tri-class categorization of brain tumours.

The tumour areas were segmented by Mohsen et al. from 2-d brain MRI imagings by Fuzzy C-mean clustering, and discrete wavelet transform (DWT) characteristics were afterwards retrieved. The compressed features have been sent to the deep neural network (DNN) which included 7 hidden classification layers. Some techniques were merely tumour detection compared with the above-mentioned research. Afshar et al., for example, have tried to solve two key CNNs issues for the problem of tumour identification, which are the necessity for huge amounts of training information and the absence of considerable transformation capability.

The researchers examined the Capsules Networks with four major goals, namely attaining maximum precision for tumour identification, examining the overfitting problem, adequacy of CapsNets for segmented tumour areas and entire MRIs for better understanding, and visualising the characteristics of MRI. For tumour identification, CapsNets perform quite well. But they are quite sensitive to the diverse backdrop of the image. Afshar et al. have developed this challenge in their follow-up study by presenting a modified model of CapsNets that examines the borders of tumour during its principal tumour identification process. 
For glioma tumour classification at high and low levels, Ge et al. presented a 3-d CNN multiscal model. They have created a feature-fusion system to better improve tumour areas by refining multiscale characteristics. In another experiment, Ge et al. employed 2-DCNN using a combination of features to improve classification performance. For the categorization of glioma in high and low grades, Decuyper et. al. has utilised pretrained VGG models and retrieved characteristics from the first fully connected layer. The deep CNNbased Glioma classification CAD system was introduced by Banerjee et al. Pereira et al. initially generated a 3-D-U-Net model from tumour areas and then transformed it to their suggested Glioma CNN grading after pictures resizing.
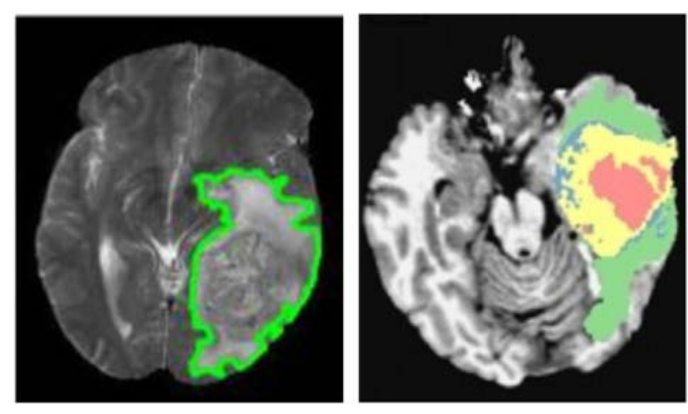

Figure 6. Image segmentation

The CNN model is proposed by global average pooling, which is a complete layer and serves as a summary of each feature map followed by a 1/1/1/1 cascade. Nevertheless, on the use of Glioma genetic algorithms, Anaraki et al. recently suggested a CNN model that was developed using three grades of Glioma genetic algorithms. Similarly, Sajjad et al. divided the tumour areas into four-degree categories by utilising deep features and then finalised a pretrained VGG-19 model. In order to expand current training data sets, they employed eight distinct data extension technologies with a total of 30 parameters. Six novel approaches in the field of tumour identification with MRI have more recently been described. The new research and all past techniques of segmentation, use, classifier, data set and objective classifications are specific information.

\section{Machine Learning in healthcare}

The objective of brain tumour diagnosis, from a clinical point of view, is to reliably detect and locate tumour tissues utilising clearly defined patient data and diagnostic characteristics from modelledMRI pictures. The right medical definition should lead to proper and prompt treatment of the condition. For this objective, clinical expertise and a database that represents high-level information where a choice and diagnoses may be made are necessary. Because of the many forms and types of tumours, manual brain tumour diagnosis is time consuming and less reliable because of over 120 recognised brain tumours.

The interest of machine learning in modern computers has been significant, and the subject of health is one of the areas of focus. Different new learning approaches have been applied in the area of brain tumour diagnostics. In order to simplify the usage of brain pictures and to increase the information collected, for instance, sophisticated techniques such as image denoise, image reconstruction, skull striping, registration were employed. This has opened the possibility to build semi-automatic, and then completely automated systems for tumour detection with greater accuracy and decreased processing time, for the cooperation of doctors, technologists and computer engineers. 
In automated diagnosis of brain tumours, radiologists and doctors have often to start the procedure, examine the result or corrected mistakes in the conclusion of the process manually. Computerized tools using previous knowledge and intelligence are nevertheless utilised to undertake tumour diagnostic procedures without human contact in a completely automated brain tumour diagnosis. In order to study the accuracy and processing time machine learnings can achieve in the diagnosis of tumours, the Machine Intelligence Research Center for Psychotic Disorders and a Research Team of the Capital Medical University conducted a 225-case competition for the diagnosis of the brain tumour to determine manual accuracy and machine diagnostics.

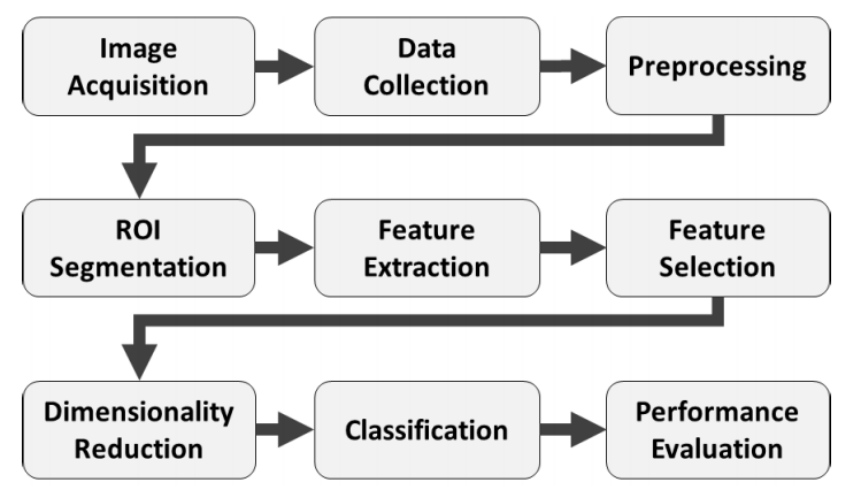

Figure 6. Various stages of Tumor detection with DNN

In a machine diagnosis, 195 accurate diagnoses were obtained with a ratio of 87 per cent in 15 minutes, on the one hand. On the other hand, a team of 15 radiologists has identified 148 patients properly, with a ratio of $66 \%$ in 30 minutes. These results indicate the higher level of accuracy, time and effort of radiologists using machine learning diagnosis over manual diagnostic. An example of how four independent specialists manually divide the brain tumour on a single MRI and the same patient. Although approaches of semi-automatic diagnostics and fully-automatic diagnostics have improved outcomes than physical analysis, manual diagnosis is utilised to identify tumour samples in clinical trials. This review research is regarded an additional step in encouraging radiologists and engineers to collaborate in the development of precise and efficient diagnostic tools for the brain tumour based on machine or technical knowledge. However, this problem was not thoroughly addressed in the baseline and most common CNN architecture.

In addition, a single data set was used for tests and confirmation of tumour detection results in several of the current research. The authors examined CapsNets for BTC for example, but only presented a single data set. For these reasons, the baseline and latest CNN models for tumour identification using various data sets must be investigated. In this regard, the section uses two data sets to compare different state-of-the-art fortumor CNNs, i.e. "Data set 1" and "Data set 2." The other four sets of data just are not accessible or contain 3-D pictures which differ from the first two datasets and thus cannot be analysed by the CNNs. AlexNet, GoogleNet, VGG, SqueezeNet, MobileNet and ResNet are utilised for the comparison.

The machine that has already been utilised to construct these CNNs has a TITAN X (Pascal) GPU from NVidia GetForce. We also utilise NVidia DIGITS for the assessment of Caffe deep learning framework. In each model's training phase, multiple alternatives on 
different parameters are explored because of the variability of the output accuracy. These factors include the number of times that positively impact the accuracy to a particular level. We have chosen 30 epochs according to the present training configuration because the accuracy after this limit stops growing. Another essential parameter is the kernel function which, after rigorous tests, is chosen as the 0.001 optimum. The next affected parameter is the kind of solver which, because of its improved performance, had been picked for stochastic gradient descent. The last argument is the loss function Softmax which calculates the Softmax classification's multinomial logistic loss.

\section{MRI for Brain Analysis}

MRI is considered to be a non-invasive method using non-ionizing and safe radiation that shows the anatomical three-dimensional structure to any body part without opening the area. The pictures may be acquired using RF pulses and a high magnetic field. The goal is to position the body within a powerful magnetic field. At first, the magnets are off and the water molecules in the body are in balance. The magnets will be switched on to the magnetic field. The water molecules of the body are aligned inside the direction of the magnetic field under the effect of this high magnetic field.

A powerful RF energy pulse is used to generate a spin against magnetic strength and a reorientation in the direction of the magnetic field. The water molecules reach their balance position when the RF energy pulse is shut off and again align with the magnetic field. The water molecules therefore transmit RF energy that the scanner detects and converts to displayed pictures. The quantity of RF power produced by the molecules of water depends on the structure of the tissue. By changing the scanner parameters, the strength of the RF radiation produced may be changed, allowing numerous model pictures to be generated through the MRI machine. The brain can indeed be represented in several ways. MRI, CT, SPECT and PET are these modalities.

Currently, MRI is the most significant imaging method for medical imaging in use. It has several benefits over other imaging modes, including the safety and rich provision of information, as the four MRI modalities retrieved from the 2013 BRATS database show. MRI was created in 1970.MRI is famous for its numerous benefits, which allow physicians to recognise structural brain abnormalities. It is costly and not appropriate for people with claustrophobia, though. A collection of 2D pictures can represent a 3D brain volume during MRI image capture. The diagnostic role of each REM modality is distinct. T2 pictures describe edematous areas. In T1 scans, healthy tissues may be seen. Images with T1-Gd are utilised for tumour boundary differentiation.

In images with fluid attenuated reversal recovery (FLAIR), edemate areas can be differentiated from Cerebrospinal Fluid (CSF). Various forms of contrast pictures can be utilised to generate the contrasts between images obtained by different MRI modes. For diagnosis, four conventional MRI methods are applied: FLAIR, MRI T2 (T2), MRI (T1) T1 (weighed), and MRI T1 (weighted by gadolinium) (T1-Gd). Each approach must be verified in order to assess its efficiency by comparing a quantitative index to a true model. In general, experts create a truth model. Expert radiologists and physicians can also assess new technologies or use simulated pictures. 
The McConnell Neuroimaging Center has created BrainWeb; an anatomical model of the normal and diseased brainsare provided via an MRI simulator. BrainWeb offers a variety of non-uniformity intensity levels ("RF") (0, 20 and 40, each of which has diameter of $1 \mathrm{~cm}$, $15 \mathrm{~cm}, 5,15 \mathrm{~cm}$, and $10 \mathrm{~cm}$ with varying noise levels (for example $0 \%$ ), 1\%, 5\%, 7\%, and 9\%. The Center for Morphometric Analysis has established the Internet Brain Segmentation Repository. The MRI database of Brain contains files with 16-bit integer formats structured as 256 v256. The Harvard Medical School AANLIB data base has six major parts: a neuroimaging first and divisions on facial skeleton, a neoplastic disease, a degenerative illness and an inflammatory disease.

BIC Analytics is utilised for the development of computer based image analysis methodes for diagnosis of brain disorders (such as Alzheimer disease, Schizophrenia, autism, and traumatic brain injury) by the Center for Biomedical Image Computing and Analytics at the University of Pennsylvania. These databases have been utilised to address the problems of computing medical image and computer assistance. The Cancer Imaging Archive has established the Image Database for the Assessment of Therapy Response. It comprises imagery information collected from 19 patients. The Neuroimaging Initiative for Alzheimer's disease includes two forms of Alzheimer's and includes imaging data gathered from 400 individuals. Allen brain atlas offers the normal picture dataset with picture data from 20 cases.

Moreover, "Brain-development.org" offers a dataset from three hospitals for normal patients. Different methods of medical imaging have been introduced in recent years to help the doctors determine the kind and location of the disease. The imaging method also allows physicians to estimate their general well-being and survival. Some of the procedures now utilised to identify anomalies in any body area are CT-scanning, X-rays, MRI, MRS, PPET scanning, and so on. In the sense that MRI is a non-invasive $3 \mathrm{D}$ imaging method that can identify the abnormality extremely well in Soft Tissues or non-bony areas, it has attracted great attention and acclaim. It contrasts best in tissue structure, since it can display pictures down to 65535 background pixels that cannot be viewed with a naked eye.

The MRI equipment may acquire many pictures from various perspectives of the individual under investigation. The brain tumour is essentially an uncontrollable massstructure of living and dead cells within the brain. The survival rate for brain tumour patients depends on the size and gravity of the disease. There are two forms of brain tumour: primary and secondary tumour, depending on their source. Primary brain tumours occur within the brain, while secondary tumours form inside the body and subsequently move to the brain. Brain tumour is categorised as benign and malignant according to its severity.

They develop slowly, seem normal, and have regular borders while malignant tumours are aggressive, in that they can risk their lives because their growth is very quick, and they are quite irregular. They are less severe since they can be life threatening. The World Health Organisation (WHO) has categorised malignant tumours in four separate classes, taking into account the tumor's chemical and physical characteristics. In virtually all of the research quoted in this report, MRI multimodality was universally recognised as a standard imagery approach for detecting brain cancers since it is very capable. Nearly 70 percent of researchers initially treat MRI pictures to improve and remove noise. 
In combination with the preparation phase, some writers additionally employ data growth to enlarge the data set. Subsequently the region of the tumour is segmented utilising any existing image processing techniques. Following classification, the partitioned zone is supplied for training purposes as input to a Deep Learning system. Another often used approach is to insert pre-processed pictures straight into the Deep Learning system without segmentation. Segmentation is not a key classification component since most people do not utilise it for classification purposes. From the input picture and the training on these features the algorithm achieves the features. Ultimately, the training process is characterized.

Even though the extraction of features in deep learning algorithms is not necessary, research has demonstrated that machine learning or metaheuristic techniques are still utilised as hybrid models to incorporate efficient and robust classification features. The characteristics are retrieved by use of statistical tools or learning methods. Then the Deep Learning algorithm is trained using this feature vector. The last technique observed in the literature is to immediately introduce the brain MRI pictures to the Deep Supervised learning without pre-classification processing. Each technique is primarily aimed at modifying the levels of both the Deep Neural network to meet the experimental requirement and then select the model that delivers the best possible results.

Hybrid models have also been utilised for the construction of efficient systems by concatenating machines and profound learning models. The data set is trained separately, testing and validation sets before any of the above stated operations are started. In Deep Learning, Convolutional Neural Network (CNN) received much appreciation and recognition in the sense that it can extract deep features automatically by adjusting to tiny picture change. It is also able to manage large quantities of data. CNN's identification and categorization of brain cancers utilising RMD has produced extremely difficult outcomes to date.

\section{Deep Learning Approaches}

Deep learning is the process of a convolutionary neural network with many layers, which has numerous hidden levels and free parameters. Unlike the conventional neural network with several levels, every MRI image is passing through a succession of convolution layers with filters (kernels), bagging layers and fully connected (FC) layers. Classical machine learning as well as profound learning are instruments for artificial understanding; but deep learning is more effective than standard machine learning. Such features include a huge variety of existing variables that provide a solution to any relationship, hidden-level functions and complicated layer-by- layer linkages.

Each layer can further execute various structure- and type-specific activities. Notwithstanding the benefits of deep learning, typical limitations such as the necessity to create a complicated architecture with hidden layers, the high cost of computing during training, and the great quantity of data necessary to achieve desired performance in the training process remain. In addition, these drawbacks increase the training time. The machine learning network architectures include several different kinds and names, such as CNN, deep residual (DRN) network, deep feeding network (DFF), DCIGN (deep inverse graphical network), deep believing network (DBN) (DN).

In the realm of image processing the CNN architecture is the most prevalent. It includes primarily of an input layer; extraction layers using convolutionary layers, a linear 
unit rectified layer (ReLU) as an activation function, pooling layers and classification layers. Deep learning methods, particularly CNN, have grown their popularity among scientists in the diagnosis of brain images, resulting in a preference to take a deep learning over standard machine learning approaches. A CNN understand the point the complicated characteristics of the brain MRI pictures itself, allowing the study to focus on network construction, not the collection and minimization of information. Patches generated from microarray Datasets are supplied as inputs to $\mathrm{CNN}$, and for the extraction of representative and complicated characteristics local sub-sampling and coagulative filters are utilised. From the findings collected, the greatest framerate and model size owing to efficiency is attained using SqueezeNet.

In most situations, however, the model is overcrowded, meaning that the data sets are entirely skewed towards one class. Nearly comparable findings were obtained by AlexNet and GoogleNet but owing to their critical nature not enough for consideration in CAD. In addition, MobileNet's and ResNet's findings are poor and their application in real-world BTC systems is not reliable enough. The results reveal that VGGNet achieved the greatest accuracy with a similar average fps but greater model size compared to any other CNNs being taken into account. Both industries and clinics have visibility into the findings, which means that they may choose a means of their choosing, taking their needs, accuracy, deployment conditions and other limitations into consideration.

The processing and analysis of brain tumour MRI data is one of the most difficult challenges for computer vision specialists. MRI is a state-of-the-art method to provide excellent pictures of human body components. It plays an essential part in the treatment and detection of the proper stage and the suitable treatment for the individual with tumour. Researchers have put forward several automated approaches with MRI to achieve this goal (T1, T2, and FLAIR). The major reason is that such RMIs are not harmed by radiation and are better off in comparison to other methods.

In medical image analysis this is taken into account, as various aspects, including similitude action, modality, content of images, transform, implement and optimise algorithm, impact on performance. Also, the selection of a BTC machine learning technique is an important stage that has to be well evaluated. Most machine learning approaches use imageextracted features, followed by their categorization, using conventional Strategys. If the retrieved characteristics are high-dimensional, this procedure might be too complicated and time intensive. The complexity of classes and the difficulty connected with distance measuring between pictures are further concerns linked to machine learning techniques. It can also be shown that medical pictures are generally impacted by low light contrast, which degrades their quality and hence lowers the accuracy of the categorization.

Techniques depending on deep learning, notably CNNs for tumour identification issues have recently gained popularity at the cost of handmade function-based methods. CNN-based approaches have numerous problems, notwithstanding their strength and enormous popularity. For example, they need a great deal of training data, which either cannot be made available for each domain and the necessary precision for a target problem might be very hard to achieve. Moreover, an increase in layers of a CNN model cannot ensure an improvement in the accuracy of classification. 
Conversely, deep learning algorithms are computer-cost because of the gear they run under. This remains an issue that is not resolved by the use of these models in real settings, particularly in clinical practise. In conclusion, there are numerous recommendations and future guidelines for research scientists to address the problems the present research community faces. The priority in the treatment of health issues with CAD approaches is to achieve better precision. The studies of BTC's literature are fuller, although certain topics require additional investigation.

\section{Brain tumor detection models}

Many professionals in the area of medical tomography and sensitive treatment have achieved significant progress in brain tumour diagnosis in recent years. The proposal was both completely automated and semi-automatic. Clinical diagnostic strategy acknowledgement was based on simple computation and supervisory levels. Diagnoses of brain cancer are separated into procedures of tumour detection, segmentation and classification, detailed in full in the next section. The reporting approaches are also provided with performance comparisons. Detection is the detection of tumours with MRI image databases or lack of them.

Recognition is among the most frequent medical issues. A MRI is a normal or an aberrant picture of the tumour detection procedure. MRI may be used in brain tumour identification utilising several approaches like SVM, ANN, KNN, as well as FFBPNN. The most frequent approaches employed are outlined and various detection techniques based on the characteristic type, detection methodology and performance are evaluated. To achieve the best detection technique, the classifier is trained and the ideal way is used for extracting features and detection is a big standard database.

For brain tumour identification using MRI, several machine learning approaches and various methodologies are available. DWT is used to get 1024 feature and PCA to decrease to 7 using the technique provided by El-Dahshan et al. The detection step employs an ANN of three layers, seven terminals in the input layer, four synapses in the hidden layer, and one single neural in the output layer is then given with the 7 features. Two classifiers are employed in the detection phase for the feedback artificial neural (FP-ANN) and KNN back propagation networks. There are 60 aberrant and 10 ordinary MRI pictures in your database.

In MRI pictures and distinguishing normal from abnormal images, Zhangetal et al. proposed a BPNN technique for detecting brain cancers. The transforming wavelet is used to remove features from the pictures and then PCA is used to minimise feature dimensions. A 3level Haar Wavelet decomposition has also been employed to decrease the features recovered from 2048 to 1024 and the levels 3 coefficients approximated. In all, the BPNN is trained by 19 major components. The structure of the neural network is: 19 input nodes, 10 hidden layer neurons, and one output layer neuron. The dataset contains 66 pictures, including 48 images that are aberrant and 18 that are normal.

The decreased functionality is transferred to the BPNN classification system. Saritha et al. suggested a new approach for the normal or abnormal classification of brain MRI imaging. Wavelet web plots are used for the extraction of three functions. The detection technique is utilised using a PNN. The PNN consists of two layers: the aggressive layer and the base layer. There are 75 pictures in the dataset: 15 regular and 60 aberrant photographs. A 
combination of 52 pictures is utilised to test and 23 pictures. For early brain tumour identification, Yang et al. developed a novel method for MRI. To extract features, a 3-level 2D-DWT has hair wavelets. Optimizing on the basis of biogeography is used.

As an RBF function classifier, a kernel-type SVM is utilised. The collection includes 90 T2 RMI pictures with a total of 256 to256 pixels, of which 5 are normal or 85 are aberrant. A three-level energy wave method reduces the characteristics from 65,536 to just 10 . $\mathrm{H}$. Kalbkhani et al. employed 2D DWT to model a detailed sub-band utilising generalised selfrepressive conditional heteroskedasticity to reduce wavelet characteristics to 24 characteristics by 61.440 . In order to extract features, linear discrimination analyses are performed, and PCA reduces the vector characteristics.

The eight-class scenario has seven functionalities, while a two-class scenario selects one function. Finally, the detection procedure involves a KNN and an SVM identificator. There are 70 abnormally and 10 ordinary MRI pictures in the database. The training package comprises 21 unnatural and 3 normal pictures, and 49 unnatural and 7 normal pictures. For tumour detection, Xuan and Liao presented a technique. Three types of features, including intensity, symmetry and texture-based characteristics, are retrieved from the input picture. 40 characteristics are then chosen, including 1 feature based on symmetry, 13 features based on intensities and 26 features based on texture. The features are derived from a variety of picture formats with 9 features retrieved from T1 pictures, 12 from T1-weighted images, and 19 from FLAIR images.

In the detecting phase, an AdaBoost technique is employed. Statistics for 10 patients with three MRI volumes are included in this collection. There are 24 slices for every volume. The data collection is also split into workouts and test sets. DWT and Daubechys wavelets were employed by Othman et al. to extract MRI imaging features, generating 17,689 vectors per picture. The tumour detection with kernel-type RBF is then applied to an SVM. Sindhumol S. et al. introduced a strategy to identify brain tumour by RIM based on an independent component analysis of spectrum clustering. Spectronically, MRI images are divided into many clusters, and ICA is utilised in conjunction with an SVM. There are 20 unusual and 60 typical MRI pictures in the database.

Abdullah et al. subsequently employed the DWT for pre-processing and extracting pictures and for creating 17,689 vectors per image. A SVM is then used to recognise normal or abnormal retrieved characteristics. A novel technical detection was suggested by Nandpuru et al. In the pre-processing step, a median filter can remove noise. Dilatation and erosion methods are then used for skull mashing on the MRI image and grayscales, symmetry and texture are used to extract the characteristics. PCA is utilised to minimise the number of features, which an SVM subsequently uses during detection, from 28 to 24 features. In all, the SVM is formed using 46 brain MRI pictures, and in the test phase 50 MRI images.

Chandra et al. suggested a novel brain tumour identification technique using pictures of MRI using a PSO-based clustering algorithm. The method discovers the centres of several classes, selects the best worldwide and changes the cluster centres depending on the highest Euclidean average distance. There are 110 aberrant and 62 regular MRI pictures in the database. The novel technique for brain cancer detection has been created and presented by Machhale et al. The system includes the pre-processing of images, extraction and 
identification of functions. During preprocessing, a morphological operation and skull masking is used.

The feature extraction step extracts symmetry, grayscale and texture and 28 characteristics from each MRI picture. During the detection step, KNN, SVM and a hybrid identity are utilised. The dataset includes 96 brain MRI pictures, 46 training images and 50 test images. Another Najadat et al. study developed a technique for detecting brain tumours. The technique extracts brain MRI pictures. In order to identify the brain MRI picture as normal or pathological, ANN and KN N methods are employed. There are 615 aberrant and 95 standard MRI pictures in the database. There are 278 characteristics that are retrieved by texturing from the MRI picture.

In a research by Kharrat et al., the following five steps were also given with a different methodology: DWT and spatial grey dependency matrix extract characteristics. Simulated annealing for vector reductions is then used, reducing 13 extracted functions to 7 chosen functions. The GA-SVM model and the stratified k-fold cross validations are used to optimise the parameters that are subsequently sent to the SVM identifier. The dataset includes 83 MRI pictures in the brain: 22 training images (10 aberrant and 12 normal) and 61 test images (44 abnormal and 17 normal).

A method for evaluating the association between the tumour and the brain's lateral ventricles was proposed by Xiao et al. for segmenting the tumour using MRI images. The technique has four phases: pre-processing, extraction of features, segmentation and detection. In order to detect aberrant pictures they utilise KNN and traditional clusters. A novel method for brain tumour detection using RIM was proposed by Ibrahim et al. PCA is used to decrease the dimensionality of the picture characteristics to 64 detective features. Subjects with normal or abnormal pictures may be identified using a BPNN with the following structure: a 65-node input layer, a 10-neuron hidden layer, and an output layer of 64 neurons.

Deepa and Devi suggested a collection, recognition and simulation procedure. Using a statistical approach, optimal texture characteristics are removed from the picture examined. In the detection and segmentation stages a BPNN and radial base function (RBF) neural network is employed. This data set includes 42 brain MRI pictures (300 abnormal and 21 good), 31 training pictures and 12 test pictures. A hybrid approach for brain tumour identification using MRI was introduced by Mohsen et al. The picture is pre-processed with a neural network with a feedback pulse. Discreet transformation of the wavelet and PCA are used to extract and reduce features.

The LL subband data towards the PCA as feature vectors is supplied by a two-level DWT decomposition. PCA is then used to generate a vector with 7 characteristics to detect. The picture is then classified as normal or abnormal in the MRI image. The system contains three layers with both the following scheme: 7 input layer nodes, 5 hidden layer neurons and one output layer neuron. In a system termed the hybrid neuro-fuzzy system, Goswami and Bhaiya coupled a neural network with the fugitive logic in order to identify the existence of brain malignancies.

\section{Challenges and Research Scope}

In the medical image processing field, brain tumours are still a prominent area of investigation. A complete overview of the latest methods for the diagnosis of brain tumours 
was given in this article. The detection of tumours is the method of detection by MRI scans of the presence or lack of brain tumours. The result would be that a number of pictures need to be examined further by the segmentation and categorization of tumours. Tumor classification means a tumour categorization procedure based upon tissue examination as benign or malignant, HG or LG or as a certain type of tumour.

The majority of the approaches examined focus on tumour diagnostic semi-automatic and automated methods. Moreover, most of them employ methods for preprocessing, feature extraction, reduction of features, segmentation and classification. The noise must be minimised since most algorithms are sensitive to noise. In the medical image processing field, brain tumours are still a popular study issue. The present study provided a thorough evaluation of the most advanced brain tumour diagnostic methods. The method of detection of tumours is the identification by MRI scans of the presence or absence of brain tumours.

The result of the detection procedure is that a number of pictures require additional analysis through the segmentation and categorization of tumours. Tumor classification is a tumour classification technique that is based on tissue examination as malignant or benign, HG and/or LG or tumour type. Most of the approaches examined focus on semi-automatic and automatic tumour diagnostic methods. Most of them also utilise methods of preprocessing, extraction of functions, reduction of functions, segments and classification. The noise must be minimised since most algorithms are sensitive to noise.

The deep learning paradigm recently shown excellent performance in the analyses of medical images, particularly for the identification of brain tumours. Deep neural networks have attained more precision than traditional techniques to machine learning. Additionally, in contrast to classical machine learning using complicated methods, the performance of deep learning networks is increased. Traditional approaches to master learning involve complicated methods of extraction and reduction of functions, whereas in deep learning techniques are not required. Deep learning methods are a time-consuming procedure that is highly data-hungry. This approach may thus need tool parallel processing to speed up the training process for big data sets, for example graphical processing units.

Although profound learning performs better, standard machine teaching approaches still have few benefits. The most common benefits of conventional algorithms are the easyto-understand and-interpret mechanisms, with less time and hardware computational costs required for their structures as well as lower computer resources for training and validation. Generally speaking, this big MRI database supports proper assessments and reliable technologies and methods utilised in the various phases of the diagnosis of brain tumours using MRI pictures. The development of a big data base is also vital for competitive development to provide improved tools and generalised approaches to fulfil your objectives with enough MRI images.

The use of standard databases provides the assumption that a fair comparison and a uniform implementation environment will enhance the performance and applicability of CAD approaches. Although numerous contributions were documented in this study focusing separately on the three major phases of tumour diagnostics, there were no full diagnostic systems comprising of all three diagnostic phases. The absence of a comprehensive diagnostic system inside one package presents two emerging issues: a lack of a completely 
automated process, because certain procedures need to be done manually, and a lack of integration between the three processes.

The development of a comprehensive and automatically system is intended to facilitate the brain tumour diagnostic procedure for physicians and radiologist. The excitement in deep learning had also led to intensive research efforts in the last several years in this family of learning machines, resulting in innumerable applications, such as the one in this overview. However, several difficulties still not properly addressed in the application of deep learning approaches. Renowned practical precautions include the architectural design of profound learning models, slow back gathering for highly complex deep architectures, their perceived absence of readability, or the cumbersome phase of tuning of hyperparameters required to perform such models optimally in a given task.

The scientific community's general recognition of these difficulties has shifted its attention on using combinatorial optimization and the swarm intelligence to hybridise deep learning models with aspects from other fields of computer intelligence. In fact, an increasing variety of research concentrate on various flavours of bio-inspired heuristics of optimization in order to address intrinsic difficulties arising from deeper learning. For example, using various types of evolutionary computation has led to tools to automatically build and configure large neural networks, similar to a new rebirth of ancient notions that cross neural and evolutionary computing. In the same line, bio-inspired heuristics, which are especially dedicated to major global optimization concerns, may now be considered an important competing player in the traditional gradient backpropagation algorithm that dominates the range of deep architecture training algorithms.

More effective transfer of know-how across diverse categorization tasks is part of further crossovers within these two fields which can be a catalyst in the medical field, allowing the use of deep learning models in new situations with data scarcity. We believe that deep learning models enabled for evolutionary computation, swarm intelligence and FA, as in any other specific field of application, will expedite and increase the quality of its outcomes while facing emerging problems in multimedia brain tumour detection and characterisation. Newcomers to this discipline should focus their efforts on this perhaps rewarding avenue of research.

\section{Conclusion}

Numerous researchers have helped identify tumours, but there remain many obstacles. Therefore, in a single survey we carried out this study in order to offer a comprehensive documentation on profound learning BTC techniques and bring academic and industry attention to the essential improvement in this field. This article reviewed all techniques for deep learning of tumour identification with their accomplishments and limitations extensively and provided full information with their relevant resources on the current publicly available data sets. Different Diagnostic methods for BRI pictures were examined in this study. A comparison research was also carried out on the basis of limits and performance indicators of classical machine learning and deep education. Different data bases and criteria in the three stages have been found in the study. Standard tumour segmentation and classification databases are employed; however, no standard tumour detection databases are available. All approaches utilised to identify a tumour are standard ways of machine learning; however, the application of deep learning methods in these processes is predicted to provide good 
outcomes. Their advantages are yet not fully utilised in the brain tumour investigation. A thorough evaluation can be inferred from the aforementioned study that there is a great demand for a fully automated unified framework that effectively detects and classifies the brain tumour into several fewer complex classifications.

\section{References}

1. Abiwinanda, M. Hanif, S. T. Hesaputra, A. Handayani, and T. R. Mengko, 'Brain Tumor Classification Using Convolutional Neural Network’, in IFMBE Proceedings, Springer, Singapore, 2019, pp. 183-189.

2. Afshar, K. N. Plataniotis, and A. Mohammadi, 'Capsule Networks' Interpretability for Brain Tumor Classification Via Radiomics Analyses’, in 2019 IEEE International Conference on Image Processing (ICIP), 2019, pp. 3816-3820.

3. Ahmad, K. Muhammad, M. Y. Lee, and S. W. Baik, "Endoscopic image classification and retrieval using clustered convolutional features,” J. Med. Syst., vol. 41, no. 12, p. 196, Dec. 2017.

4. Ali, A. R. Syafeeza, L. J. Geok, Y. C. Wong, N. A. Hamid, and A. S. Jaafar, 'Design of Automated Computer-Aided Classification of Brain Tumor Using Deep Learning', Springer, Singapore, 2019, pp. 285-291.

5. Denys et al., "The processing of visual shape in the cerebral cortex of human and nonhuman primates: A functional magnetic resonance imaging study,” J. Neurosci., vol. 24, no. 10, pp. 2551-2565, Mar. 2004.

6. Deepak and P. M. Ameer, 'Brain tumor classification using deep CNN features via transfer learning', Comput. Biol.Med., vol. 111, no. March, p. 103345, 2019, doi: 10.1016/j.compbiomed.2019.103345.

7. Gumaei, M. M. Hassan, M. R. Hassan, A. Alelaiwi, and G. Fortino, “A hybrid feature extraction method with regularized extreme learning machine for brain tumor classification,” IEEE Access, vol. 7, pp. 36266-36273, 2019.

8. Hossain, F. S. Shishir, M. Ashraf, M. A. Al Nasim, and F. Muhammad Shah, 'Brain Tumor Detection Using Convolutional Neural Network’, 1st Int. Conf. Adv. Sci. Eng. Robot. Technol. 2019, ICASERT 2019, vol. 2019, no. Icasert.

9. Kalbkhani H, Shayesteh MG, Zali-Vargahan B. Robust algorithm for brain magnetic resonance image (MRI) classification based on GARCH variances series. Biomed. Signal Process. Control 2013;8(6):909-19. https://doi.org/10.1016/j.bspc.2013.09.001.

10. Li, L. Kuang, S. Xu, and Z. Sha, 'Brain Tumor Detection Based on Multimodal Information Fusion and Convolutional Neural Network', IEEE Access, vol. 7, pp. 180134-180146, 2019, doi: 10.1109/ACCESS.2019.2958370.

11. Muthu Krishnammal and S. Selvakumar Raja, 'Convolutional neural network based image classification and detection of abnormalities in MRI brain images', Proc. 2019 IEEE Int. Conf. Commun. Signal Process. ICCSP 2019, pp. 548-553, 2019.

12. Pereira, A. Pinto, V. Alves, and C. A. Silva, "Brain tumor segmentation using convolutional neural networks in MRI images,” IEEE Trans. Med. Imag., vol. 35, no. 5, pp. 1240-1251, May 2016.

13. Saritha M, Joseph KP, Mathew AT. Classification of MRI brain images using combined wavelet entropy based spider web plots and probabilistic neural network. 
Pattern Recogn. Lett. 2013;34(16):2151-6. https://doi.org/10.1016/j.patrec.2013.08.017.

14. Sengupta, C. B. McNabb, N. Kasabov, and B. R. Russell, "Integrating space, time, and orientation in spiking neural networks: A case study on multimodal brain data modeling,” IEEE Trans. Neural Netw. Learn. Syst., vol. 29, no. 11, pp. 5249-5263, Nov. 2018.

15. Sultan, N. M. Salem, and W. Al-Atabany, 'Multi-Classification of Brain Tumor Images Using Deep Neural Network', IEEE Access, vol. 7, pp. 69215-69225, 2019, doi: 10.1109/ACCESS.2019.2919122.

16. Tang, S. Ahmad, P.-T. Yap, and D. Shen, "Multi-atlas segmentation of MR tumor brain images using low-rank based image recovery,” IEEE Trans. Med. Imag., vol. 37, no. 10, pp. 2224-2235, Oct. 2018.

17. Wang, S. Du, A. Atangana, A. Liu, and Z. Lu, “Application of stationary wavelet entropy in pathological brain detection,” Multimedia Tools Appl., vol. 77, no. 3, pp. 3701-3714, Feb. 2018.

18. Xuan X, Liao Q. Statistical structure analysis in MRI brain tumor segmentation. Fourth International Conference on Image and Graphics (ICIG 2007) Aug 2007. p.421-6. https://doi.org/10.1109/ICIG.2007.181.

19. Yang G, Zhang Y, Yang J, Ji G, Dong Z, Wang S. Automated classification of brain images using wavelet-energy and biogeography-based optimization. Multimed. Tools Appl. May 2015;26:1-17. https://doi.org/10.1007/s11042-015-2649-7.

20. Zhou et al., 'Holistic Brain Tumor Screening and Classification Based on DenseNet and Recurrent Neural Network', Springer, Cham, 2019, pp. 208-217.

21. Zhang Y, Dong Z, Wua L, Wanga S. A hybrid method for MRI brain image classification. $\quad$ Exp. $\quad$ Syst. Appl. 2011;38(8):10049-53. https://doi.org/10.1016/j.eswa.2011.02.012. 